\title{
Exclusive $J / \psi$ production in ultraperipheral collisions at the LHC: constraints on the gluon distributions in the proton and nuclei
}

\author{
V. Guzey and M. Zhalov \\ National Research Center "Kurchatov Institute", Petersburg Nuclear Physics Institute (PNPI), \\ Gatchina, 188300, Russia \\ E-mail: vguzey@pnpi.spb.ru, zhalov@pnpi.spb.ru
}

\begin{abstract}
Using the framework of collinear factorization in perturbative QCD (pQCD), we analyze the recent data of the LHCb and ALICE collaborations on exclusive photoproduction of $J / \psi$ in ultraperipheral $p p$ and $A A$ collisions, respectively. We demonstrate that the simultaneous analysis of the proton and $\mathrm{Pb}$ data allows us to reduce the ambiguity of the pQCD description of the $\gamma p \rightarrow J / \psi p$ and $\gamma A \rightarrow J / \psi A$ cross sections and, hence, to place additional constraints on the gluon distributions in the nucleon and nuclei at small $x$. We also make predictions for the cross section of coherent exclusive photoproduction of $J / \psi$ in nucleus-nucleus ultraperipheral collisions accompanied by the electromagnetic excitation of nuclei and the subsequent neutron emission.
\end{abstract}

KEYWORDS: ultraperiphereal collisions, nuclear shadowing, gluon distribution in nuclei and in the proton 


\section{Contents}

1 Introduction 1

2 UPCs at the LHC and exclusive $J / \psi$ photoproduction in pQCD 2

2.1 Exclusive $J / \psi$ photoproduction on the proton and nuclei in LLA of pQCD 2

2.2 LO pQCD description of $J / \psi$ photoproduction on the proton 6

2.3 LO pQCD description of $J / \psi$ photoproduction on Lead 8

3 Discussion and conclusions

\section{Introduction}

Recently the LHCb [1] and ALICE [2,3] collaborations presented results of their measurements of exclusive $J / \psi$ photoproduction in ultraperipheral proton-proton and nucleusnucleus collisions at the LHC, respectively. These results are of particular interest because the analysis of exclusive $J / \psi$ photoproduction within the leading logarithmic approximation of perturbative QCD (pQCD) predicts [4-7] that the cross section of this process is proportional to the gluon density of the target squared. Since the fraction $x$ of the target momentum carried by the gluons is inversely proportional to the beam momentum, at the high energies achievable at the LHC, it is potentially possible to obtain unique information on the small $x$ behavior of the gluon density in the proton and nuclei and, thus, to constrain the current ambiguity in this quantity.

The LHCb collaboration measured the yield of $J / \psi$ at forward rapidities $(2<y<4.5)$ in proton-proton ultraperipheral collisions (UPCs) at $7 \mathrm{TeV}$ [1]. This data allowed one to extract the $\gamma p \rightarrow J / \psi p$ cross section in two regions of the $\gamma p$ center-of-mass energy $W_{\gamma p}$ : (i) $0.4 \mathrm{TeV}<W_{\gamma p}<1.3 \mathrm{TeV}$ corresponding to the values of $x=M_{J / \psi}^{2} / W_{\gamma p}^{2}$ in the range of $6 \times 10^{-6}<x<6 \times 10^{-5}$, which significantly extends the region of small $x$ studied at HERA, and (ii) $15 \mathrm{GeV}<W_{\gamma p}<51 \mathrm{GeV}$ corresponding to $0.004<x<0.04$, which is covered by earlier high-precision fixed-target experiments. The analysis [1] confirmed the power law energy dependence of the $\gamma p \rightarrow J / \psi p$ cross section $\left(\sigma\left(W_{\gamma p}\right) \propto W_{\gamma p}^{\delta}\right.$ with $\delta=0.92 \pm 0.15)$ consistent with the previous HERA results and did not reveal any evidence of new phenomena such as the onset of the gluon saturation regime at small $x$.

The ALICE collaboration measured coherent $J / \psi$ photoproduction in ultraperipheral $\mathrm{PbPb}$ collisions at $\sqrt{s_{N N}}=2.76 \mathrm{TeV}$. The cross section was measured in two regions of the rapidity of produced $J / \psi$ : in the range of $-3.6<y<-2.6[2]$ and at central rapidities of $-0.9<y<0.9$ [3]. The $\gamma P b \rightarrow J / \psi P b$ cross section obtained in the data analysis was compared $[2,3]$ to different model predictions and the better agreement was found with models including moderate nuclear gluon shadowing (the predictions referred to as RSZ-LTA [8] and AB-EPS09 [9], see figure 3 of [2] and figure 5 of [3]). 
In this article, using the framework of collinear factorization of pQCD and the leading logarithmic approximation (LLA), we extend and continue the analysis of $J / \psi$ photoproduction in heavy ion UPCs [10] and calculate the $\gamma p \rightarrow J / \psi p$ and $\gamma P b \rightarrow J / \psi P b$ cross sections employing various parameterizations of the gluon distribution in the proton and $\mathrm{Pb}$ and compare them to the data mentioned above. We argue that the simultaneous description of the proton and $\mathrm{Pb}$ data is (i) possible, (ii) points to sizable nuclear gluon shadowing at $x=10^{-3}$ and below, and (iii) places constraints on the value of the hard scale $\mu^{2}$ probed in coherent $J / \psi$ photoproduction, on the size of higher order corrections to the commonly used LLA formula suggested in [4-7], and, thus, on the gluon distribution in $\mathrm{Pb}$ down to $x=10^{-3}$ as well as on the gluon distribution in the proton down to $x=6 \times 10^{-6}$. These values provide an order of magnitude improvement in the small $x$ kinematic coverage compared to those achieved respectively at HERA in lepton-proton deep inelastic scattering (DIS) experiments and at fixed-target nuclear DIS experiments.

We also make predictions for the cross section of coherent exclusive photoproduction of $J / \psi$ in nucleus-nucleus UPCs accompanied by the electromagnetic excitation of nuclei and the subsequent neutron emission.

\section{UPCs at the LHC and exclusive $J / \psi$ photoproduction in pQCD}

\subsection{Exclusive $J / \psi$ photoproduction on the proton and nuclei in LLA of pQCD}

The cross section of $J / \psi$ production in symmetric nucleus-nucleus UPCs (for a review of the UPC physics, see [11]) has the following form:

$$
\frac{d \sigma_{A A \rightarrow A A J / \psi}(y)}{d y}=N_{\gamma / A}(y) \sigma_{\gamma A \rightarrow J / \psi A}(y)+N_{\gamma / A}(-y) \sigma_{\gamma A \rightarrow J / \psi A}(-y)
$$

where $N_{\gamma / A}(y)=\omega d N_{\gamma / A}(\omega) / d \omega$ is the photon flux; $y=\ln \left(2 \omega / M_{J / \psi}\right)$ is the $J / \psi$ rapidity, where $\omega$ is the photon energy and $M_{J / \psi}$ is the mass of $J / \psi$. The presence of two terms in eq. (2.1) reflects the specific feature of UPCs: each colliding nucleus can radiate a photon and can also serve as a target. Thus, at each value of the rapidity $y$ - except for the case of $y=0$ - there are two contributions corresponding to production of $J / \psi$ by low energy photons with the energy of $\omega_{L}=\left(M_{J / \psi} / 2\right) e^{-y}$ and by high energy photons with the energy of $\omega_{H}=\left(M_{J / \psi} / 2\right) e^{y}$, respectively. The separation of these two contributions can be easy realized in two cases: (i) at $y=0$, when one obtains $\sigma_{\gamma A \rightarrow J / \psi A}(y=0)=\left(d \sigma_{A A \rightarrow A A J / \psi}(y=0) / d y\right) /\left(2 N_{\gamma / A}(y=0)\right)$, and (ii) in the region of the rapidities $y$, where one of the contributions dominates and, hence, one obtains $\sigma_{\gamma A \rightarrow J / \psi A}(y)=\left(d \sigma_{A A \rightarrow A A J / \psi}(y) / d y\right) / N_{\gamma / A}(y)$. Therefore, since the photon flux $N_{\gamma / A}(y)$ can be calculated with reasonable accuracy (with the uncertainty of the order of $5 \%$ ), $J / \psi$ production in UPCs can be effectively used to study the $J / \psi$ photoproduction cross section.

At high energies and small transverse momenta $p_{t}$ of $J / \psi\left(W_{\gamma p} \gg M_{J / \psi} \gg p_{t}\right)$, using the framework of collinear factorization and in the leading logarithmic approximation (LLA) of pQCD which is usually referred to as the leading order (LO) approximation, the cross section of coherent $J / \psi$ photoproduction on the proton or a nucleus $(T=p, A)$ 
reads $[4-7,12]$ :

$$
\frac{d \sigma_{\gamma T \rightarrow J / \psi T}\left(W_{\gamma p}, t=0\right)}{d t}=\frac{M_{J / \psi}^{3} \Gamma_{e e} \pi^{3}}{48 \alpha_{\text {e.m. } \mu^{8}}}\left(1+\eta^{2}\right) R_{g}^{2} F^{2}\left(\mu^{2}\right)\left[\alpha_{s}\left(\mu^{2}\right) x G_{T}\left(x, \mu^{2}\right)\right]^{2},
$$

where $\Gamma_{e e}$ is the width of the $J / \psi$ electronic decay; $\alpha_{\mathrm{em}}$ is the fine structure constant; $\alpha_{s}\left(\mu^{2}\right)$ is the running strong coupling constant. In eq. $(2.2), G_{T}\left(x, \mu^{2}\right)$ is the density of gluons in the target $T$, where the gluons carry the momentum fraction $x=M_{J / \psi}^{2} / W_{\gamma p}^{2}$ of the target and are resolved at the hard scale $\mu$ which is of the order of the mass of the charm quark, $m_{c}$ (the precise value of $\mu^{2}$ will be discussed in section 2.2).

Equation (2.2) contains several factors taking into account intricate aspects of the discussed reaction which mostly affect the normalization of the $\gamma p \rightarrow J / \psi p$ cross section, but not so much its $W_{\gamma p}$ dependence.

- The factor of $\eta$ is the ratio of the real to the imaginary parts of the $\gamma T \rightarrow J / \psi T$ amplitude. This quantity can be evaluated using the well-known Gribov-Migdal relation $[13,14], \eta=\tan (\pi \lambda / 2)$, where $\lambda$ parametrizes the behavior of $x G_{T}\left(x, \mu^{2}\right)$ at small $x, x G_{T}\left(x, \mu^{2}\right) \propto 1 / x^{\lambda}$. Taking $\lambda=0.2$, which broadly reproduces the energy behavior of the $\gamma p \rightarrow J / \psi p$ cross section (see the discussion below), we obtain $1+\eta^{2}=1.11$.

- In the framework of collinear QCD factorization for hard exclusive processes $[15,16]$, the $\gamma p \rightarrow J / \psi p$ amplitude is expressed in terms of the gluon generalized parton distribution (GPD) rather than the usual one. One can take this fact into account phenomenologically by introducing the enhancement factor $R_{g}[14,17,18]$ :

$$
R_{g}=\frac{2^{3+2 \lambda}}{\sqrt{\pi}} \frac{\Gamma\left(\frac{5}{2}+\lambda\right)}{\Gamma(4+\lambda)} .
$$

Note that the expression for $R_{g}$ depends on the model/parameterization for the gluon GPDs and, hence, is model-dependent. To estimate the magnitude of the effect, we use $\lambda=0.2$ and obtain $R_{g}=1.18$ [14].

- Note that in our analysis, we employed the form $x G_{T}\left(x, \mu^{2}\right) \propto 1 / x^{\lambda}$ only to evaluate the factors of $\eta$ and $R_{g}$. Moreover, in the above estimates, we used $\lambda=0.2$ only for illustration. In practice, in the interval $5 \times 10^{-6} \leq x \leq 0.01$, each gluon distribution used in our work (including nuclear gluon distributions) was fitted using the shape $x G_{T}\left(x, \mu^{2}\right) \propto 1 / x^{\lambda}$ and the corresponding $\lambda$ was determined from the fit. We found that this simple fit works with a high accuracy and the resulting values of $\lambda$ can be taken constant (except for the MSTW08 gluon distribution) and are close to $\lambda=0.2$ (for details, see the Appendix and table 3 there).

In the $0.01 \leq x \leq 0.1$ interval, which corresponds to the low- $W$ and large- $|y|$ edges of the kinematics considered in our work, the gluon distributions can no longer be accurately approximated by the simple fit $x G_{T}\left(x, \mu^{2}\right) \propto 1 / x^{\lambda}$ (except for the MNRT07 gluon distribution). Therefore, the small- $x$ asymptotic expressions of eq. (2.3) and 
$\eta=\tan (\pi \lambda / 2)$ become progressively less accurate. Our analysis of $R_{g}$ based on [18] and of $\eta$ based on [19] shows that the extrapolation of our low- $x$ results to the $0.01 \leq x \leq 0.1$ interval leads to certain theoretical uncertainties which increase with an increase of $x$. Depending on the used gluon distribution, these uncertainties are, e.g., of the order of $5-10 \%$ for $R_{g}$ and $5-7 \%$ for $1+\eta^{2}$ at $x=0.05$ and of the order of $10-20 \%$ for $R_{g}$ and $10-15 \%$ for $1+\eta^{2}$ at $x=0.1$.

- The main uncertainty in eq. (2.2) comes from the suppression factor $F^{2}\left(\mu^{2}\right), 0<$ $F^{2}\left(\mu^{2}\right)<1$, which contains all effects that go beyond the leading order collinear factorization that we use: next-to-leading order corrections, the overlap between the photon and $J / \psi$ light-cone wave functions weighted by the gluon distribution of the target either in the $k_{T}$ factorization approach [6] or in the dipole formalism [7, 12, 20], corrections associated with the charmonium wave function [21], etc.

Equation (2.2) was originally derived in the non-relativistic approximation for the charmonium wave function [4] that neglects the motion of the charm quarks in $J / \psi$. In this case, $F^{2}\left(\mu^{2}\right)=1$ and $\mu^{2}=M_{J / \psi}^{2} / 4=2.4 \mathrm{GeV}^{2}$. The magnitude of relativistic and other corrections, i.e., the deviation of $F^{2}\left(\mu^{2}\right)$ from unity, and the related issue of the choice of the value of $\mu^{2}$ are subjects of discussion in the literature $[6,12,20,21]$. However, while these corrections mainly influence the absolute value of the $\gamma p \rightarrow J / \psi p$ cross section (the total uncertainty of LO pQCD predictions is estimated $[6,21]$ to be about $30 \%$ or less), its energy dependence is predicted by eq. (2.2) and is given by the $x$ dependence of $x G_{T}\left(x, \mu^{2}\right)$.

In this work, we do not attempt to evaluate $F^{2}\left(\mu^{2}\right)$ theoretically using, e.g., different models of the charmonium wave function. Instead, we adopt the following strategy. First, by comparing predictions of eq. (2.2) with the available high-energy data on the $W_{\gamma p}$ dependence of the $\gamma p \rightarrow J / \psi p$ cross section, we fix the value of $\mu^{2}$. Second, by requiring that eq. (2.2) describes also the magnitude of the $\gamma p \rightarrow J / \psi p$ cross section, we determine the normalization of the LO pQCD cross section given by eq. (2.2), i.e., we phenomenologically find $F^{2}\left(\mu^{2}\right)$.

Turning to the $t$ dependence of the $d \sigma_{\gamma p \rightarrow J / \psi p} / d t$ cross section, we note that its $p_{t}$ dependence is usually parameterized in the exponential form of $\exp \left[-B_{J / \psi}\left(W_{\gamma p}\right) p_{t}^{2}\right]$, with the slope parameter $B_{J / \psi}\left(W_{\gamma p}\right)$ weakly depending on energy. A good description of the HERA data on the $t$ dependence of the $J / \psi$ photoproduction cross section [22-25] is obtained using the following Regge-motivated parameterization of the slope $B_{J / \psi}\left(W_{\gamma p}\right)$ :

$$
B_{J / \psi}\left(W_{\gamma p}\right)=4.5+0.4 \ln \left(\frac{W_{\gamma p}}{90 \mathrm{GeV}}\right)
$$

which is consistent with the parameterizations and the values of $B_{J / \psi}\left(W_{\gamma p}\right)$ reported in $[22-$ $25]$.

Hence, for the proton target, the total $J / \psi$ photoproduction cross section is:

$$
\sigma_{\gamma p \rightarrow J / \psi p}\left(W_{\gamma p}\right)=\frac{M_{J / \psi}^{3} \Gamma_{e e} \pi^{3}}{48 \alpha_{\text {e.m. }} \mu^{8}} \frac{1}{B_{J / \psi}\left(W_{\gamma p}\right)}\left(1+\eta^{2}\right) F^{2}\left(\mu^{2}\right)\left[R_{g} \alpha_{s}\left(\mu^{2}\right) x G_{p}\left(x, \mu^{2}\right)\right]^{2},
$$


where we ignored the negligibly small effect of the minimal momentum transfer to the target.

Extending eqs. (2.2) and (2.5) to the description of $J / \psi$ production on nuclei and accounting for the transverse momentum distribution dictated by the nuclear form factor $F_{A}(t)$, one obtains [26]:

$$
\begin{aligned}
\sigma_{\gamma A \rightarrow J / \psi A}\left(W_{\gamma p}\right) & =\frac{M_{J / \psi}^{3} \Gamma_{e e} \pi^{3}}{48 \alpha_{\mathrm{e} . \mathrm{m} . \mu^{8}}}\left(1+\eta_{A}^{2}\right) F^{2}\left(\mu^{2}\right)\left[\frac{1}{A} R_{g, A} \alpha_{s}\left(\mu^{2}\right) x G_{A}\left(x, \mu^{2}\right)\right]^{2} \Phi_{A}\left(t_{\mathrm{min}}\right)= \\
& =\frac{\left(1+\eta_{A}^{2}\right) R_{g, A}^{2}}{\left(1+\eta^{2}\right) R_{g}^{2}} \frac{d \sigma_{\gamma p \rightarrow J / \psi p}\left(W_{\gamma p}, t=0\right)}{d t}\left[\frac{G_{A}\left(x, \mu^{2}\right)}{A G_{N}\left(x, \mu^{2}\right)}\right]^{2} \Phi_{A}\left(t_{\mathrm{min}}\right), \quad(2.6
\end{aligned}
$$

where $G_{A}\left(x, \mu^{2}\right)$ is the nuclear gluon density; $\Phi_{A}\left(t_{\min }\right)=\int_{t_{\min }}^{\infty} d t\left|F_{A}(t)\right|^{2}$, where $t_{\min }=$ $-M_{J / \psi}^{4} m_{N}^{2} / W_{\gamma p}^{4}$ is the minimal momentum transfer to the nucleus; $\eta_{A}$ is the ratio of the real to the imaginary parts of the $\gamma A \rightarrow J / \psi A$ scattering amplitude whose skewness effect is parameterized by the factor of $R_{g, A}$. By analogy with the free proton case (see the discussion above and eq. (2.3)), we determine the factors of $\eta_{A}$ and $R_{g, A}$ using the asymptotic small- $x$ dependence of the nuclear gluon distribution $x G_{A}\left(x, \mu^{2}\right) \propto 1 / x^{\lambda_{A}}$ : $\eta_{A}=\tan \left(\pi \lambda_{A} / 2\right)$ and $R_{g, A}=\left(2^{3+2 \lambda_{A}} / \sqrt{\pi}\right) \Gamma\left(\frac{5}{2}+\lambda_{A}\right) / \Gamma\left(4+\lambda_{A}\right)$.

In this work, we are interested in small values of $x$ probed in high-energy $J / \psi$ photoproduction experiments, $6 \times 10^{-5}<x<0.05$, where the effect of nuclear shadowing significantly reduces the nuclear gluon density $G_{A}\left(x, \mu^{2}\right)$ compared to the free nucleon gluon density $G_{N}\left(x, \mu^{2}\right)$, i.e., $G_{A}\left(x, \mu^{2}\right)<A G_{N}\left(x, \mu^{2}\right)$. Hence, within LO pQCD, the influence of the nuclear medium on coherent $J / \psi$ photoproduction on nuclei [see eq. (2.6)] is related to the nuclear gluon shadowing characterized by the factor

$$
R\left(x, \mu^{2}\right)=\frac{G_{A}\left(x, \mu^{2}\right)}{A G_{N}\left(x, \mu^{2}\right)} .
$$

In addition, nuclear shadowing tames the growth of the nuclear gluon density $G_{A}\left(x, \mu^{2}\right)$ with a decrease of $x$ and makes it slower than that of the free nucleon. Parameterizing $G_{A}\left(x, \mu^{2}\right)$ at small $x$ as $G_{A}\left(x, \mu^{2}\right) \propto 1 / x^{\lambda_{A}}$, one finds that $\lambda_{A}<\lambda_{p}\left(\lambda_{p}\right.$ corresponds to the free proton) and, hence,

$$
\kappa_{A / N} \equiv\left[\frac{\left(1+\eta_{A}^{2}\right) R_{g, A}^{2}}{\left(1+\eta^{2}\right) R_{g}^{2}}\right]^{1 / 2}=0.87-0.97<1,
$$

where the spread in the values of $\kappa_{A / N}$ originates from different $x$ dependences of various nuclear and free proton gluon distributions that we used in this work. Thus, in addition to the factor of $R\left(x, \mu^{2}\right)$, the factor of $\kappa_{A / N}$ further decreases the $\gamma A \rightarrow J / \psi A$ cross section compared to the $\gamma p \rightarrow J / \psi p$ one.

It is important to point out - and it is one of the key points of the present work - that consistency of the simultaneous LO pQCD description of the $\gamma A \rightarrow J / \psi A$ and $\gamma p \rightarrow J / \psi p$ cross sections, i.e., consistency of eqs. (2.5) and (2.6), requires the use of the same proton gluon density $G_{p}\left(x, \mu^{2}\right)$ in the forward $\gamma p \rightarrow J / \psi p$ cross section and in the evaluation of the nuclear gluon shadowing factor $R\left(x, \mu^{2}\right)$. 


\subsection{LO pQCD description of $J / \psi$ photoproduction on the proton}

The results of the calculation of the total $\gamma p \rightarrow J / \psi p$ cross section using eq. (2.5) and different gluon parton distributions in the proton (see the Appendix) are presented in figure 1. These results are compared to the available high-energy HERA data on the $\gamma p \rightarrow J / \psi p$ cross section (we only omitted the $2000 \mathrm{H} 1$ data [22] which is consistent with the more recent data), the $\gamma p \rightarrow J / \psi p$ cross section extracted from the LHCb data on protonproton UPCs [1], and the fit [10,27] to the available data on $d \sigma_{\gamma p \rightarrow J / \psi p}\left(W_{\gamma p}, t=0\right) / d t$ converted into the total cross section using the slope $B_{J / \psi}\left(W_{\gamma p}\right)$ of eq. (2.4).

In the upper panel of figure 1, the theoretical predictions of eq. (2.5) are evaluated with $F^{2}\left(\mu^{2}\right)=1$ and at $\mu^{2}=M_{J / \psi}^{2} / 4=2.4 \mathrm{GeV}^{2}$ [4] using the MNRT07 [14], CTEQ6L1 and CTEQ6L [28], MRST04 [29], NNPDF [30], and MSTW08LO [31] leading order (LO) gluon parton distributions in the proton (the curves in the figure are labeled accordingly). In the Appendix, we show these gluon distributions as a function of $x$ (figure 7 ) and also give the corresponding values of the strong coupling constant $\alpha_{s}$ entering eq. (2.5) (table 2). One can see from the panel that while the MNRT07 parameterization provides an excellent description of the data-including the region of high $W_{\gamma p}$ measured by the LHCb collaboration which was not used in the analysis of ref. [14] - all other gluon distributions fail to reproduce either the normalization of the data or both the normalization and the energy dependence of the data.

To illustrate this point, we introduce the normalization factor $\zeta$,

$$
\frac{1}{\zeta}=\frac{\sigma_{\gamma p \rightarrow J / \psi p}\left(W_{\gamma p}=100 \mathrm{GeV}\right)_{\mid \text {Eq. }}(2.5)}{81 \mathrm{mb}}
$$

which is designed to reduce the value of the theoretically calculated cross section at $W_{\gamma p}=$ $100 \mathrm{GeV}$ so that it reproduces the data at this point. One can see that $\zeta$ plays the role of the suppression factor $F^{2}\left(\mu^{2}\right)$ that we discussed above. The second column of table 1 gives the resulting values of $\zeta$ at $\mu^{2}=2.4 \mathrm{GeV}^{2}$.

\begin{tabular}{|c|c|c|}
\hline Parameterization & $\zeta\left(Q^{2}=2.4 \mathrm{GeV}^{2}\right)$ & $\zeta\left(Q^{2}=3 \mathrm{GeV}^{2}\right)$ \\
\hline CTEQ6L1 [28] & 0.28 & 0.48 \\
CTEQ6L [28] & 0.27 & 0.49 \\
MRST04 [29] & 0.27 & 0.50 \\
NNPDF [30] & 0.26 & 0.52 \\
MSTW08LO [31] & 0.17 & 0.30 \\
\hline
\end{tabular}

Table 1. The normalization factor $\zeta$ of eq. (2.9).

The lower panel of figure 1 shows the LO pQCD predictions of eq. (2.5) at $\mu^{2}=2.4$ $\mathrm{GeV}^{2}$ multiplied by the corresponding factor of $\zeta$. One can see from the figure that after the renormalization by the $\zeta$ factor, the predictions using the MRST04 and NNPDF gluon distributions correctly reproduce the energy behavior and the normalization of the $\gamma p \rightarrow$ $J / \psi p$ cross section over the considered $W_{\gamma p}$ range. At the same time, the predictions with the CTEQ6L1, CTEQ6L and MSTW08LO parameterizations fail to reproduce the energy 

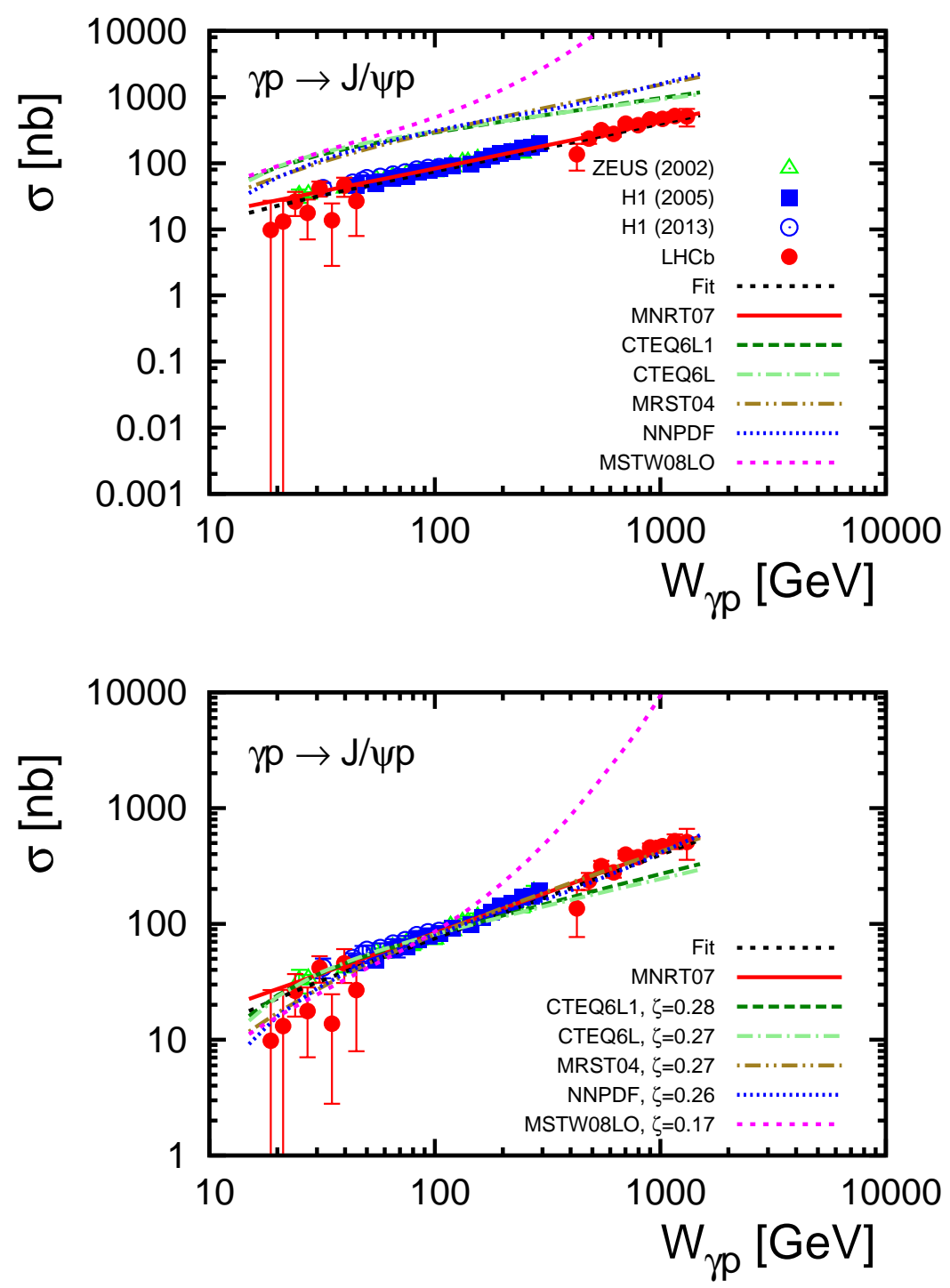

Figure 1. The $\gamma p \rightarrow J / \psi p$ cross section as a function of the $\gamma p$ center-of-mass energy $W_{\gamma p}$. Upper panel: The available high-energy data vs. the fit [10] (the curve labeled "Fit") and LO pQCD predictions of eq. (2.5) at $\mu^{2}=2.4 \mathrm{GeV}^{2}$ using various gluon distributions of the proton, see text and the Appendix. Lower panel: The same as in the upper panel, but with the pQCD predictions reduced by the multiplicative factor $\zeta=F^{2}\left(\mu^{2}\right)(2.9)$.

behavior of the $\gamma p \rightarrow J / \psi p$ cross section for $W_{\gamma p}>100 \mathrm{GeV}$ : the behavior predicted by the MSTW08LO fit is distinctly too steep, i.e., the resulting values of $\sigma_{\gamma p \rightarrow J / \psi p}$ contradict the LHCb data, and the behavior predicted by the CTEQ6L1 and CTEQ6L fits is too slow.

In addition, as we will demonstrate in section 2.3, the MNRT07 gluon distribution at $\mu^{2}=2.4 \mathrm{GeV}^{2}$ corresponds to the prediction made in the framework of the leading twist nuclear shadowing, which overestimates the nuclear suppression factor $S\left(W_{\gamma p}\right)$ at $x=10^{-3}$ (see figure 3). 
We have already mentioned above the uncertainty of the LO pQCD calculation of $\sigma_{\gamma p \rightarrow J / \psi p}$ related to the uncertainty in the choice of $\mu^{2}$ in eq. (2.5). In particular, on the one hand, the non-relativistic approximation that neglects the transverse momenta (Fermi motion) of $c$-quarks in the $J / \psi$ wave function prescribes that $\mu^{2}=M_{J / \psi}^{2} / 4=2.4 \mathrm{GeV}^{2}$ [4]. On the other hand, the scale $\mu^{2}$ cannot be reliably fixed by the LO formalism. The analyses accounting for effects that are not included in our formalism (see the discussion of the factor of $F^{2}\left(\mu^{2}\right)$ above) suggest that the appropriate hard scale of the $\gamma p \rightarrow J / \psi p$ process is $\mu^{2}>2.4 \mathrm{GeV}^{2}$.

We approach this problem phenomenologically by increasing $\mu^{2}$ in eq. (2.5) from $\mu=$ $2.4 \mathrm{GeV}^{2}$ to a certain value of $\mu^{2}$ so that eq. (2.5) correctly reproduces the energy behavior of the data in figure 1 , which corresponds to $x G_{p}\left(x, \mu^{2}\right) \propto 1 / x^{\lambda}$ with $\lambda \approx 0.2$. Note that since the cross section is proportional to $1 / \mu^{8}$, even a rather small increase of $\mu^{2}$ leads to a sizable reduction of the $\gamma p \rightarrow J / \psi p$ cross section.

For small $x$, the MSTW08LO fit corresponds to $\lambda \gg 0.2$ already at $\mu^{2}=2.4 \mathrm{GeV}^{2}$. Therefore, an increase of $\mu^{2}$ will further increase $\lambda$ and, thus, will make the description of the data by the corresponding LO pQCD calculation only worse (see the lower panel of figure 1). This forces us to conclude that the rapid increase of the MSTW08LO gluon distribution for small $x$ contradicts the consistent simultaneous description of the HERA and $\mathrm{LHCb}$ data on exclusive $J / \psi$ photoproduction on the proton (see also figure 8 in the Appendix).

Our analysis shows that we can obtain a good description of the energy dependence of the data presented in figure 1 by eq. (2.5) evaluated at $\mu^{2}=3 \mathrm{GeV}^{2}$ and this description will be equally good for all tested gluon distributions in the proton, except for MSTW08LO which has already been ruled out. This is illustrated in figure 2, where the upper panel presents predictions of eq. (2.5) at $\mu^{2}=3$ (assuming $F^{2}\left(\mu^{2}\right)=1$ ) and the lower panel presents the same predictions multiplied by the corresponding factor of $\zeta$, which plays the role of $F^{2}\left(\mu^{2}\right)$. The corresponding values of $\zeta$ at $\mu^{2}=3 \mathrm{GeV}^{2}$ are given in the third column of table 1 . One can see from the lower panel of figure 2 that the choice of $\mu^{2}=3 \mathrm{GeV}^{2}$ and $F^{2}\left(\mu^{2}\right) \approx 0.5$ in eq. (2.5) allows one to achieve the good description of the $W_{\gamma p}$ dependence and normalization of the $\gamma p \rightarrow J / \psi p$ cross section at collider energies using various sets of the gluon distribution in the proton.

Note that the MNRT07 prediction at $\mu^{2}=3 \mathrm{GeV}^{2}$ in figure 2 has been scaled by the appropriate factor to reproduce the normalization of the data - we chose not to show explicitly this normalization (the corresponding $\zeta=1.9$ ) because the normalization of this gluon distribution is obtained from a fit to the data.

\subsection{LO pQCD description of $J / \psi$ photoproduction on Lead}

It was shown in [10] that the recent ALICE measurements of exclusive $J / \psi$ production in ultraperipheral $\mathrm{PbPb}$ collisions $[2,3]$ provide direct evidence for strong nuclear gluon shadowing down to $x \sim 10^{-3}$, which can be model-independently quantified by the factor 

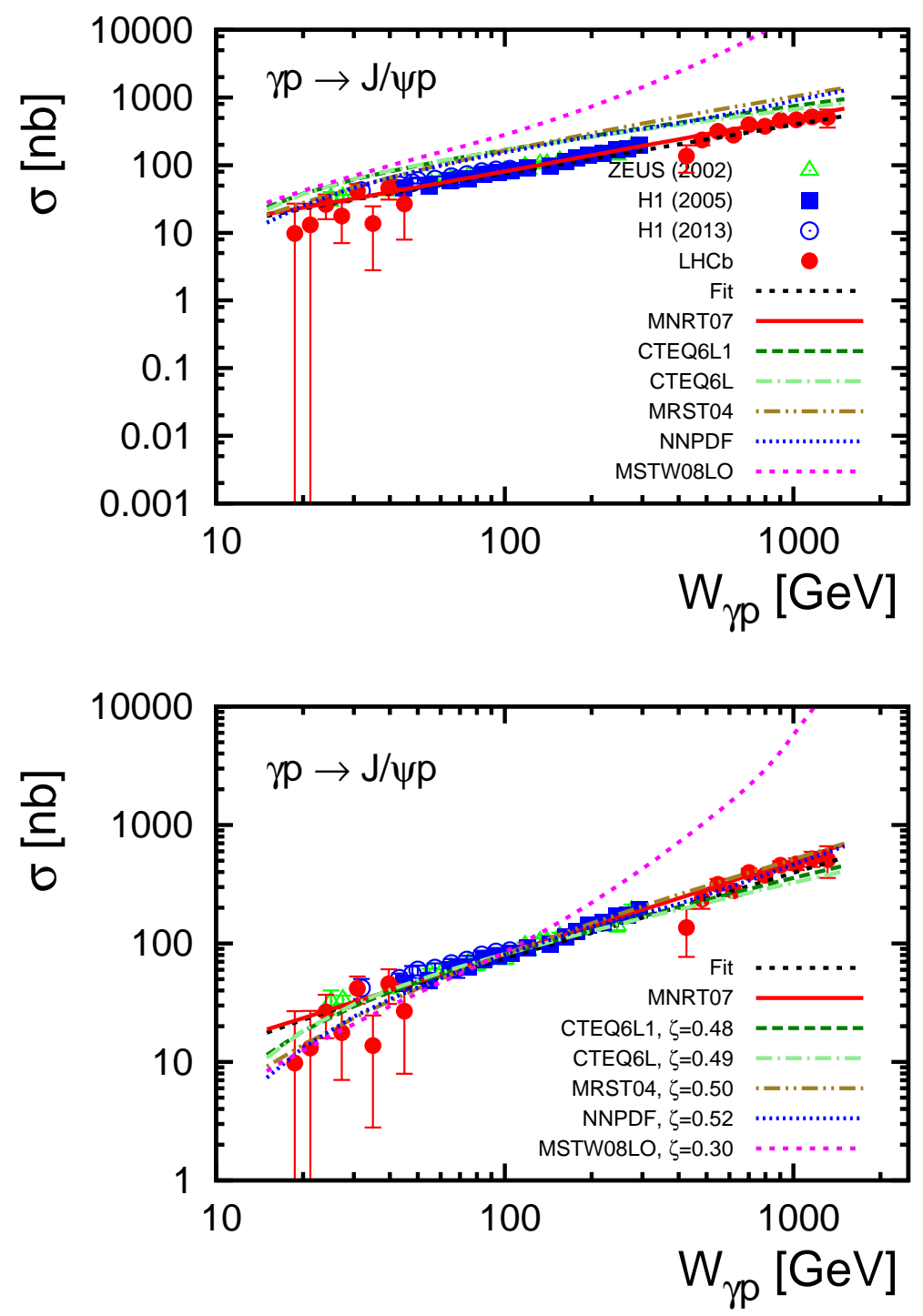

Figure 2. The same as in figure 1, but with the LO pQCD predictions evaluated at $\mu^{2}=3 \mathrm{GeV}^{2}$.

of $S\left(W_{\gamma p}\right)$ :

$$
S\left(W_{\gamma p}\right)=\left[\frac{\sigma_{\gamma P b \rightarrow J / \psi P b}^{\exp }\left(W_{\gamma p}\right)}{\sigma_{\gamma P b \rightarrow J / \psi P b}^{\mathrm{IA}}\left(\left(W_{\gamma p}\right)\right.}\right]^{1 / 2} .
$$

In eq. (2.10), the numerator is the experimental cross section and the denominator is the result of the impulse approximation neglecting all nuclear effects except for coherence, i.e., the fact that $\Phi_{A}\left(t_{\min }\right) \neq 1$. The analysis of [10] found that $S(x=0.025)=0.74 \pm 0.12$ and $S(x=0.001)=0.61 \pm 0.05$. (We used $x=M_{J / \psi}^{2} / W_{\gamma p}^{2}$.)

Combining Eqs. (2.2) and (2.6), we obtain

$$
S\left(W_{\gamma p}\right)=\kappa_{A / N} \frac{G_{A}\left(x, \mu^{2}\right)}{A G_{N}\left(x, \mu^{2}\right)}=\kappa_{A / N} R\left(x, \mu^{2}\right),
$$


where $R\left(x, \mu^{2}\right)=G_{A}\left(x, \mu^{2}\right) /\left[A G_{N}\left(x, \mu^{2}\right)\right]$ is the factor characterizing nuclear shadowing in the gluon channel and $\kappa_{N / A}$ is given by eq. (2.8).
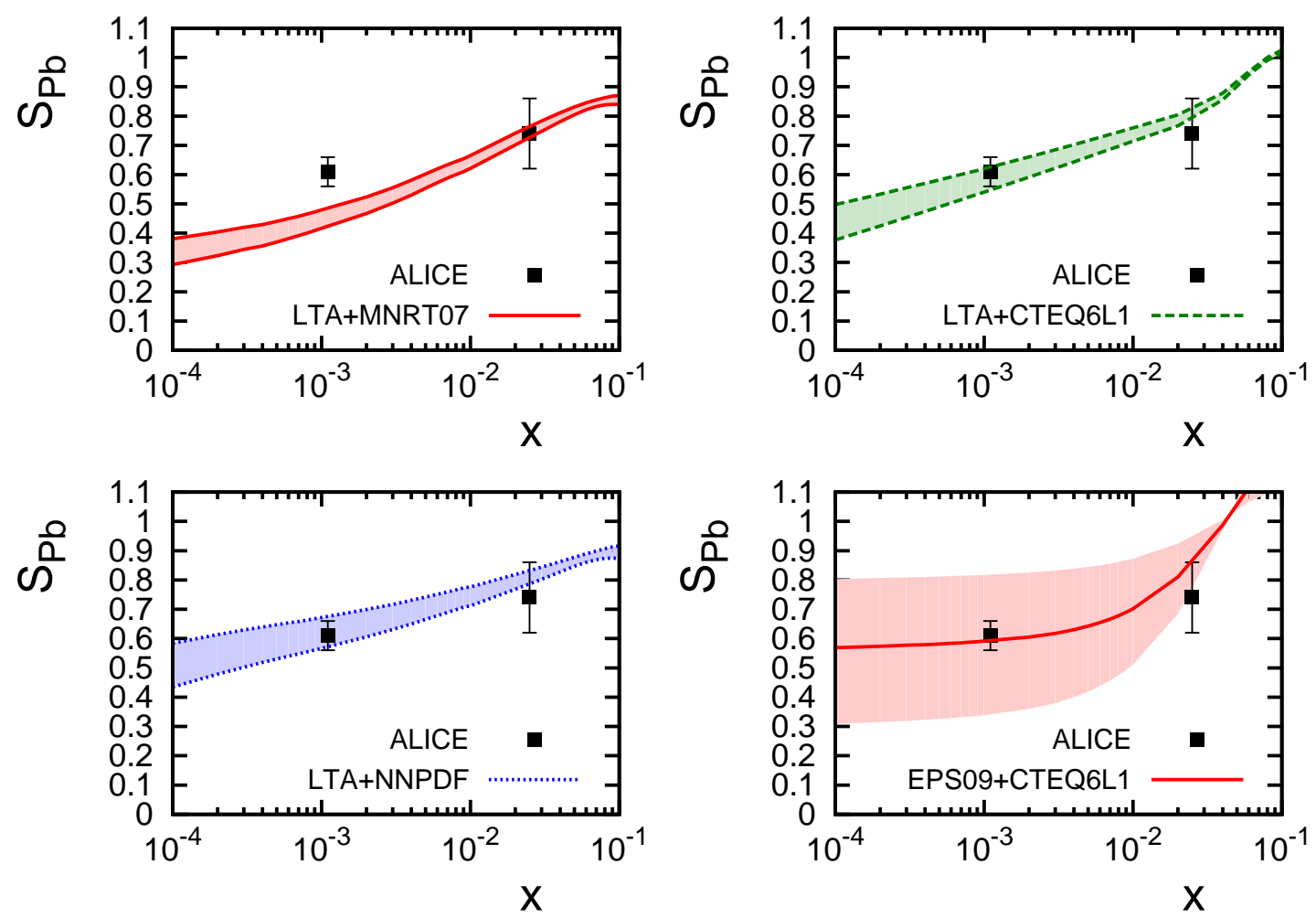

Figure 3. The suppression factor $S\left(W_{\gamma p}\right)$ for Lead of eq. (2.10) as a function of $x=M_{J / \psi}^{2} / W_{\gamma p}^{2}$. The results of the model-independent analysis of the ALICE data of [10] (labeled "ALICE") are compared with the LO pQCD predictions at $\mu^{2}=2.4 \mathrm{GeV}^{2}$ (see text for details).

Figures 3 and 4 present the suppression factor $S\left(W_{\gamma p}\right)$ for Lead as a function of $x=$ $M_{J / \psi}^{2} / W_{\gamma p}^{2}$. The results of the model-independent analysis of the ALICE data of [10] (labeled "ALICE") are compared with the LO pQCD predictions given by eq. (2.11) at $\mu^{2}=2.4 \mathrm{GeV}^{2}$ (figure 3) and at $\mu^{2}=3 \mathrm{GeV}^{2}$ (figure 4). In the two upper panels and in the lower left one, the factors of $R\left(x, \mu^{2}\right)$ and $\kappa_{A / N}$ are calculated in the framework of the leading twist approximation (LTA) consisting in the combination of the leading twist theory of nuclear shadowing [32] with the given (MNRT07, CTEQ6L1, CTEQ6L, MRST04 and NNPDF) gluon distributions of the free nucleon. In each case, we show the band of predictions which corresponds to the intrinsic uncertainty of the leading twist theory of nuclear shadowing ${ }^{1}$. Note also that since the predictions with the CTEQ6L1 and CTEQ6L and with the MRST04 and NNPDF gluon distributions are rather close, we show only the representative examples of CTEQ6L1 and NNPDF.

\footnotetext{
${ }^{1}$ The bands shown in figures 3 and 4 represent the theoretical uncertainty of the leading twist theory of nuclear shadowing [32] associated with the ambiguity in the magnitude of the contribution describing the interaction of the virtual photon with three and more nucleons of the nucleus. The upper and lower boundaries of the bands correspond to the lower and higher limits on shadowing.
} 

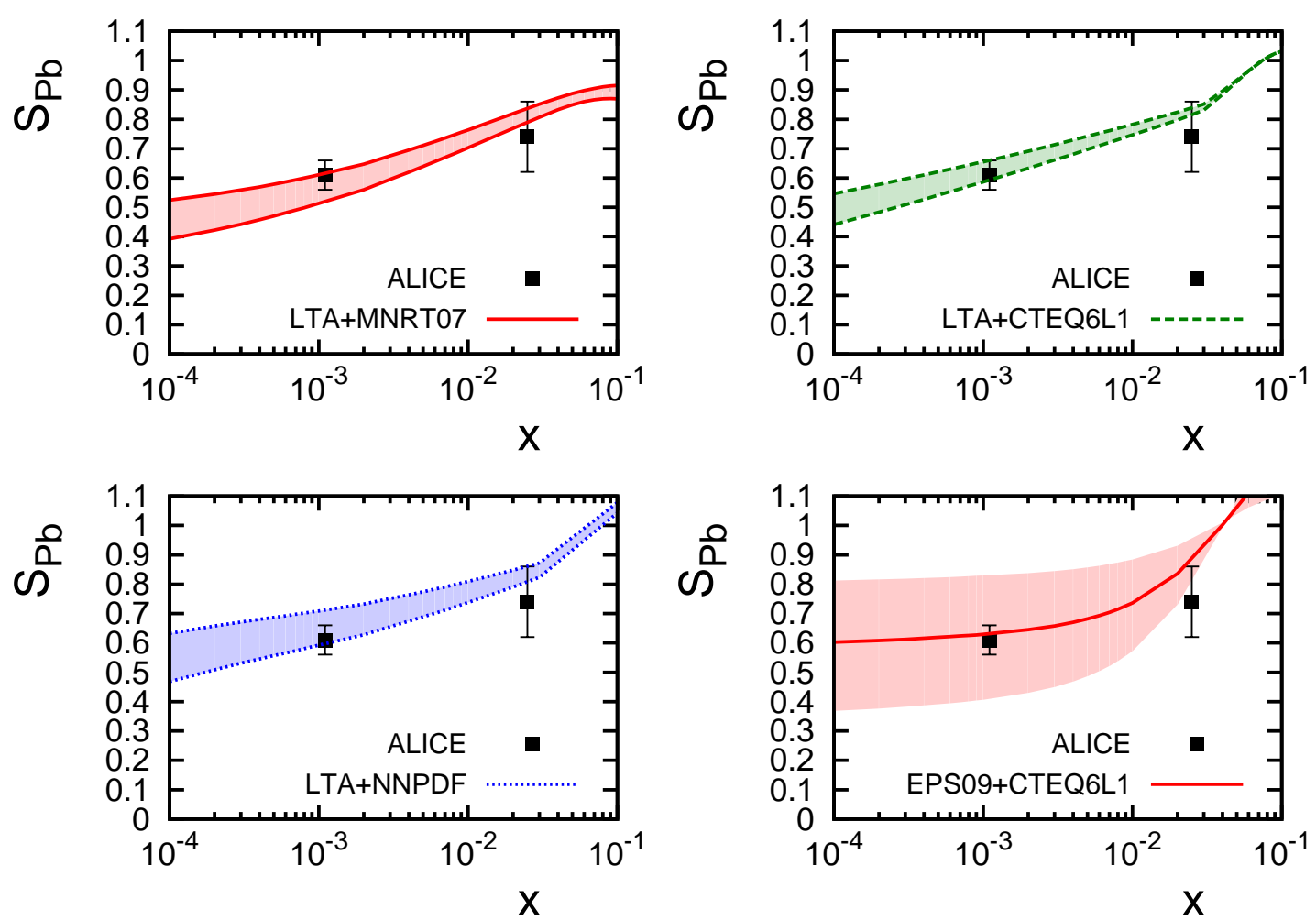

Figure 4. The same as in figure 3 , but with the LO pQCD predictions evaluated at $\mu^{2}=3 \mathrm{GeV}^{2}$.

In the lower right panels, $S\left(W_{\gamma p}\right)$ is calculated using the leading order EPS09 parameterization of nuclear PDFs [33] extracted from the global QCD fit to available data; at the leading order, EPS09 should be coupled with the CTEQ6L1 gluon distribution of the free proton. Note that we use EPS09 as a typical representative example - predictions for $S\left(W_{\gamma p}\right)$ can also be made using other parameterizations of nuclear PDFs obtained using the global QCD fits [34-36] (see also figure 3 of ref. [10]).

One can see from figures 3 and 4 that - with the exception of the case of the MNRT07 gluon distribution at $\mu^{2}=2.4 \mathrm{GeV}^{2}$ at small $x$ - the predictions of LTA [32] and of the global QCD analysis of nuclear PDFs [33] provide a very good description of the ALICE point at $x=10^{-3}$ and a fair description of the point at $x=0.025$ (keeping in mind theoretical uncertainties and experimental errors $)^{2}$.

Our results for the suppression factor $S\left(W_{\gamma p}\right)$ can also be presented in the form of the $\mathrm{PbPb} \rightarrow \mathrm{PbPbJ} / \psi$ cross section of exclusive photoproduction of $J / \psi$ in symmetric $\mathrm{PbPb}$

\footnotetext{
${ }^{2}$ One should point out that at $x=0.025$, the considered predictions of nuclear gluon shadowing converge (become essentially indistinguishable) and are all consistent with the suppression factor $S\left(W_{\gamma p}\right)$ for Lead determined with large experimental errors. At the same time, the value of $S\left(W_{\gamma p}\right)$ at $x=10^{-3}$ can in principle better discriminate between different scenarios of nuclear shadowing.
} 
UPCs (see the discussion in [10]). Combing Eqs. (2.1), (2.6) and (2.11), we obtain

$$
\begin{aligned}
\frac{d \sigma_{A A \rightarrow A A J / \psi}(y)}{d y} & =N_{\gamma / A}(y) S^{2}(y) \frac{d \sigma_{\gamma p \rightarrow J / \psi p}(y, t=0)}{d t} \Phi_{A}(y) \\
& +N_{\gamma / A}(-y) S^{2}(-y) \frac{d \sigma_{\gamma p \rightarrow J / \psi p}(-y, t=0)}{d t} \Phi_{A}(-y) .
\end{aligned}
$$

In eq. (2.12), the first term is evaluated at $W_{\gamma p}(y)=2 \sqrt{\omega_{H} E_{A}}=\sqrt{2 M_{J / \psi} E_{A}} e^{y / 2}$, where $E_{A}$ is the energy of the nuclear beam per nucleon $\left(E_{A}=1.38 \mathrm{TeV}\right.$ for symmetric $\mathrm{PbPb}$ collisions at $\left.\sqrt{s_{N N}}=2.76 \mathrm{TeV}\right)$; the second term is evaluated at $W_{\gamma p}(-y)=2 \sqrt{\omega_{L} E_{A}}=$ $\sqrt{2 M_{J / \psi} E_{A}} e^{-y / 2}$.

Our predictions for the $d \sigma_{A A \rightarrow A A J / \psi}(y) / d y$ cross section of eq. (2.12) as a function of the rapidity $y$ of $J / \psi$ at $\sqrt{s_{N N}}=2.76 \mathrm{TeV}$ are presented in figure 5. In eq. (2.12), the factor $S\left(W_{\gamma p}\right)$ and the cross section $d \sigma_{\gamma p \rightarrow J / \psi p} / d t$ are evaluated at $\mu^{2}=3 \mathrm{GeV}^{2}$ (see the discussion above). One can see from the figure that the LO pQCD formalism provides a good description of the two ALICE data points at $y=-3.1[2]$ and $y=0$ [3].
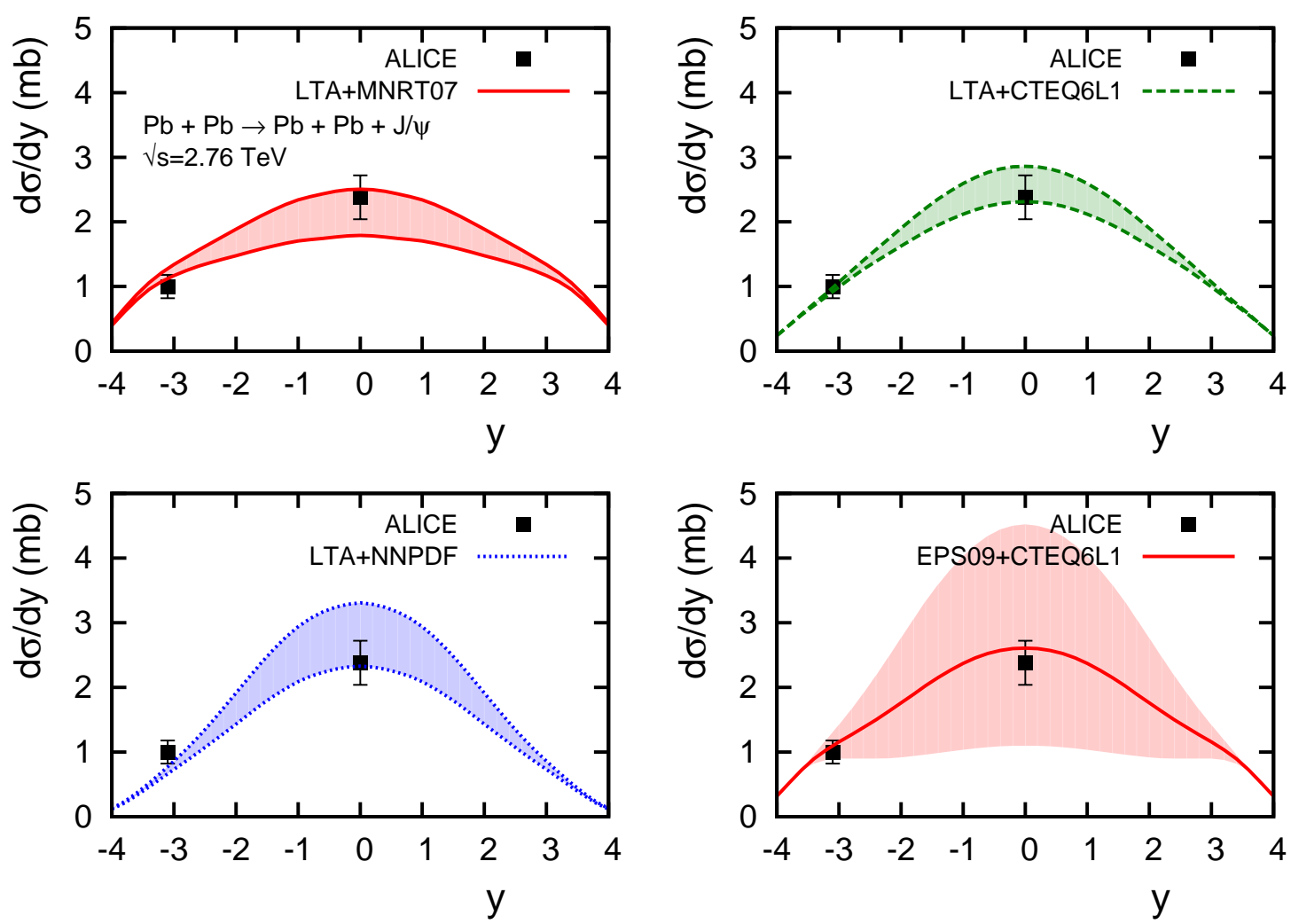

Figure 5. The $\mathrm{PbPb} \rightarrow \mathrm{PbPbJ} / \psi$ cross section of exclusive $J / \psi$ photoproduction in symmetric $\mathrm{PbPb}$ UPCs as a function of the rapidity $y$ of $J / \psi$ at $\sqrt{s_{N N}}=2.76 \mathrm{TeV}$. The ALICE data points at $y=-3.1[2]$ and $y=0[3]$ are compared with the LO pQCD predictions of eq. (2.12) at $\mu^{2}=3$ $\mathrm{GeV}^{2}$.

At the LHC, ALICE, ATLAS, the CMS and LHCf experiments are equipped with zero degree calorimeters (ZDC) allowing one to detect very forward particles produced in beam 
fragmentation. This allows one to study coherent exclusive photoproduction of $J / \psi$ in nucleus-nucleus UPCs which is accompanied by electromagnetic excitation of the nuclei with the subsequent emission of a number of neutrons by one or by both nuclei [8]. One can distinguish several channels: 1n1n corresponds to the emission of one neutron by each ion; XnXn - to the emission of several neutrons; 0n1n and 0nXn - to the excitation of only one of the ions; $0 \mathrm{n} 0 \mathrm{n}$ is the case when neither of the ions emitted a neutron. The partial cross section for each channel is calculated using eq. (2.12) with the modified flux of equivalent photons corresponding to the given channel [8].

Figure 6 presents our predictions for the partial cross sections of the 0n0n, 0nXn, $\mathrm{XnXn}$ and $0 \mathrm{n} 1 \mathrm{n}$ channels of the $\mathrm{PbPb} \rightarrow \mathrm{PbPbJ} / \psi$ reaction with neutron emission at $\sqrt{s_{N N}}=2.76 \mathrm{TeV}$. The curves are LO pQCD predictions using the leading twist nuclear shadowing and the MNRT07 gluon distribution at $\mu^{2}=3 \mathrm{GeV}^{2}$.
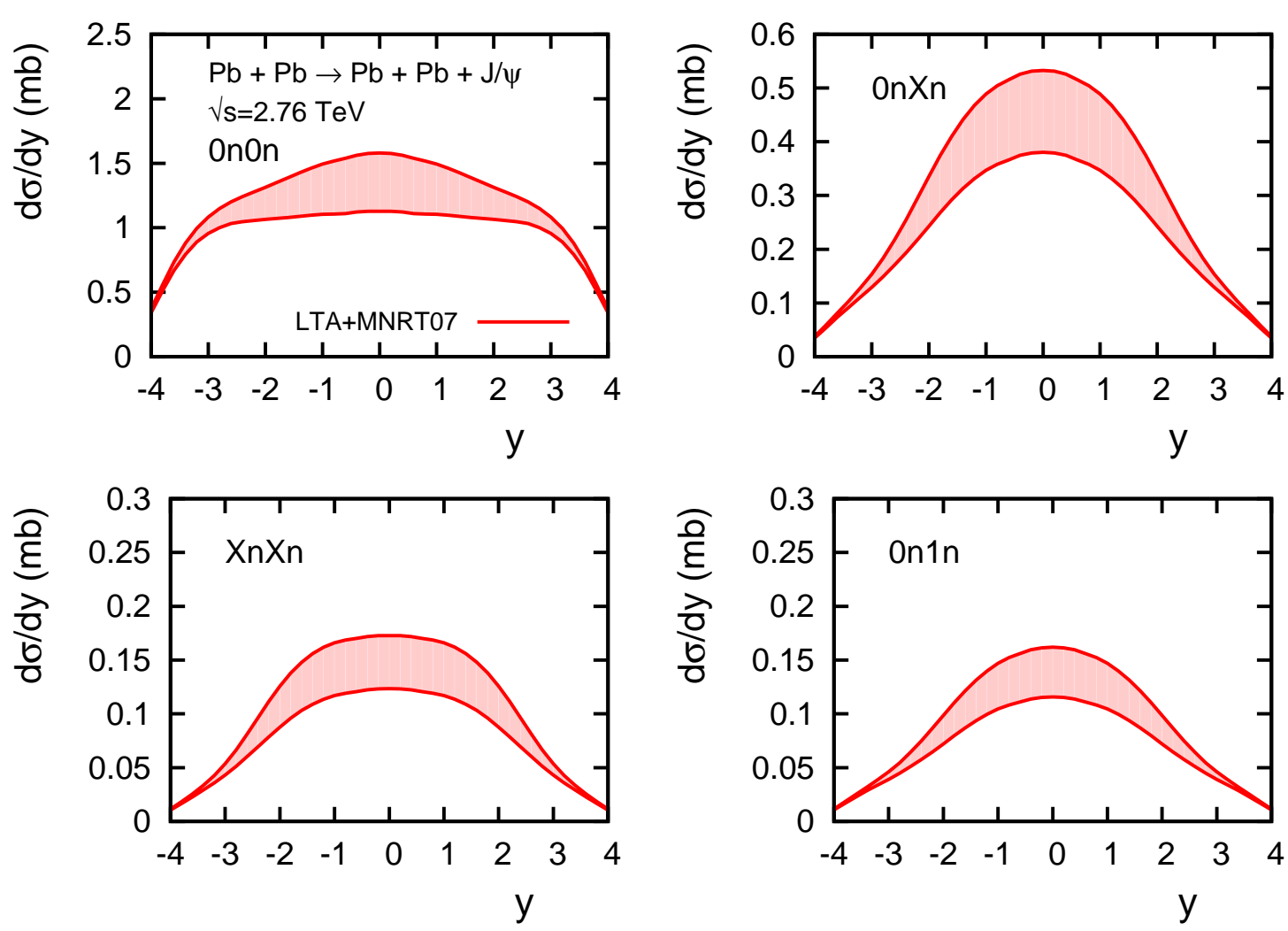

Figure 6. The partial $\mathrm{PbPb} \rightarrow \mathrm{PbPbJ} / \psi$ cross sections of exclusive $J / \psi$ photoproduction in $\mathrm{PbPb}$ UPCs at $\sqrt{s_{N N}}=2.76 \mathrm{TeV}$ accompanied by neutron emission (see text for details). The curves are LO pQCD predictions using the leading twist nuclear shadowing and the MNRT07 gluon distribution at $\mu^{2}=3 \mathrm{GeV}^{2}$.

In summary, using various sets of gluon distributions in the proton and predictions for the effect of nuclear gluon shadowing, we demonstrated that the leading logarithmic approximation provides the good and simultaneous description of the high-energy data on the $\gamma p \rightarrow J / \psi p$ and $\gamma P b \rightarrow J / \psi P b$ cross sections (see figures 2 and 4) and on the cross section of exclusive $J / \psi$ photoproduction in $\mathrm{PbPb}$ UPCs (see figure 5 ). 


\section{Discussion and conclusions}

Using the leading order pQCD, we made predictions for the $\gamma p \rightarrow J / \psi p, \gamma P b \rightarrow J / \psi P b$ and $\mathrm{PbPb} \rightarrow \mathrm{PbPbJ} / \psi$ cross sections employing various parameterizations of the gluon distribution in the proton and $\mathrm{Pb}$ and compared them to the available LHC data on proton-proton and $\mathrm{PbPb}$ UPCs. We demonstrated that the simultaneous description of the proton and $\mathrm{Pb}$ data is possible and requires sizable nuclear gluon shadowing. We found the following constraints on the description of the $\gamma p \rightarrow J / \psi p$ and $\gamma P b \rightarrow J / \psi P b$ cross sections within the LO pQCD formalism: (i) the probed effective scale is $\mu^{2} \approx 3 \mathrm{GeV}^{2}$ (for many sets of contemporary gluon distributions in the proton) and (ii) the suppression factor parameterizing a host of effects beyond the used approximation is insignificant, $F^{2}\left(\mu^{2}\right) \approx 0.5$. Thus, within the used formalism, our analysis helps to place additional constraints on the gluon distribution in the proton down to $x=6 \times 10^{-6}$ and on the gluon distribution in $\mathrm{Pb}$ down to $x=10^{-3}$. This is an order of magnitude improvement in the small $x$ kinematic coverage compared to that of lepton-proton DIS at HERA and of fixed-target nuclear DIS, respectively.

Note that while we determine the value of $\mu^{2}$ from a comparison to the data, one can still offer its physical interpretation. The fact that we found that $\mu^{2}>M_{J / \psi}^{2} / 4>m_{c}^{2}$ means that in addition to the charm quark mass $m_{c}$, the transverse momentum of the charm quarks in the $J / \psi$ wave function, $k_{t}$, also gives a noticeable contribution to $\mu^{2}$.

We also make predictions for the cross section of coherent exclusive photoproduction of $J / \psi$ in nucleus-nucleus UPCs accompanied by the electromagnetic excitation of nuclei and the subsequent neutron emission.

Our findings can be compared with the results of other approaches to exclusive photoproduction of $J / \psi$ in proton-proton and nucleus-nucleus UPCs at the LHC available in the literature.

(i) Using the framework that is similar to the one used in our paper, i.e., LO pQCD, Adeluyi and Bertulani [9] (see also [37-39]) evaluated the $\gamma p \rightarrow J / \psi p$ cross section using the MSTW08LO gluon density at $\mu^{2}=M_{J / \psi}^{2} / 4=2.4 \mathrm{GeV}^{2}$. The additional normalization factor of $\zeta_{V}=1 / 3.5=0.286$ taking into account effects beyond the used approximation had to be introduced to explain the normalization of the $\gamma p \rightarrow J / \psi p$ cross section [37]. However, as we explained above (see figures 1 and 2), the growth of the MSTW08LO gluon distribution at small $x$ is too steep (see figure 8 of the Appendix), which means that the corresponding pQCD expression fails to simultaneously describe the HERA and LHCb data on the $W_{\gamma p}$ behavior of the $\gamma p \rightarrow J / \psi p$ cross section.

In conjunction with the nuclear gluon shadowing factor $R\left(x, \mu^{2}\right)$ obtained in the global QCD analyses of nuclear PDFs (HKN07, EPS08 and EPS09), the same gluon distribution (MSTW08LO) was also used to calculate the $\gamma A \rightarrow J / \psi A$ cross section [9]. Such a procedure violates the consistency of the LO pQCD calculations in the following sense. The HKN07 nuclear PDFs [34] are based on the MRST98 parton distributions of the free nucleon [40]; the EPS09 and EPS08 LO nPDF analysis - on the CTEQ6L1 free nucleon PDFs. Hence, to be consistent, the use of the MSTW08 gluon density in the proton in conjunction with the HKN07, EPS08 and EPS09 gluon shadowing factors requires introduction of large 
$x$-dependent corrections ( $x$-dependent normalization factors given by the inversed square of ratio of the gluon distributions presented in figure 8 of the Appendix), which were not included in the predictions for the $P b P b \rightarrow P b P b J / \psi$ cross section presented in ref. [9].

(ii) Predictions for the $\mathrm{PbPb} \rightarrow \mathrm{PbPbJ} / \psi$ cross section were also made using the framework of the color dipole model $[41,42]$. The resulting calculations predict too little nuclear gluon shadowing and, as a result, overestimate the $\mathrm{PbPb} \rightarrow \mathrm{PbPbJ} / \psi$ cross section, see the discussion in [3] and [10]. Qualitatively, these results can be readily understood: on average, the elementary dipole-nucleon cross section for the $\gamma p \rightarrow J / \psi p$ process is rather small due to squeezing of the dipole by the $J / \psi$ wave function. Hence, multiple rescatterings of this dipole on the target nucleons constitute essentially a higher twist correction to the impulse approximation and, hence, cannot lead to significant nuclear attenuation (shadowing).

(iii) Exclusive coherent $J / \psi$ photoproduction in heavy ion UPCs at LHC energies was also considered in ref. [43] using the dipole formalism with the $k_{T}$-unintegrated gluon distribution. In the case of the dipole-nucleus scattering, besides the Glauber-type rescattering on the target nucleons, the approach also takes into account the small- $x$ evolution of dipoles, i.e., gluon-fusion corrections associated with the rescattering of the $Q Q g$ dipole. The net resulting nuclear shadowing correction for the process and kinematics of interest is similar to our result (the shadowing correction predicted in [43] is $15-20 \%$ smaller than our prediction).

(iv) In the framework of the dipole formalism, an attempt to take into account the contribution of a broad range of dipole sizes, i.e., the contribution of both perturbative and non-perturbative QCD contributions, to the $\gamma p \rightarrow J / \psi p$ cross section was made in [7, $12,20]$. It was estimated that the $\gamma p \rightarrow J / \psi p$ process probes the effective scales $\mu^{2}>2.4$ $\mathrm{GeV}^{2}$ and that the relativistic corrections are large, i.e., the factor of $F^{2}$ is small. While the latter differs from our finding that $F^{2} \approx 0.5$ which is consistent with the analysis of [6], one has to keep in mind that the gluon distributions used in different analyses are different which naturally results in different $F^{2}$ factors. The extension of the analysis to the nuclear case predicts sizable suppression of the $\gamma A \rightarrow J / \psi A$ cross section due to the gluon nuclear shadowing [7].

In the near future, there will be available results of measurements of $J / \psi$ photoproduction from the proton-lead run at $\sqrt{s_{N N}}=5.02 \mathrm{TeV}$. The new data will mostly probe the photon-proton interaction, which, in turn, will influence the precision of the analysis of the photon-nucleus cross section [44].

\section{Acknowledgements}

The authors would like to thank M. Ryskin and M. Strikman for illuminating discussions of their respective results on photoproduction of $J / \psi$ on the proton. 


\section{Appendix: Leading order gluon distributions in the proton and corre- sponding $\alpha_{s}$ used in the present work}

For the calculation of the perturbative $\gamma p \rightarrow J / \psi p$ cross section, see Eqs. (2.2) and (2.5), we used six different leading order (LO) gluon distributions in the proton: MNRT07 [14], CTEQ6L1 [28], CTEQ6L [28], MRST04 [29], NNPDF [30] and MSTW08LO [31]. (Note that these PDFs are listed in the order of an increasing magnitude of $x G_{p}\left(x, Q^{2}\right)$ at $x=$ $5 \times 10^{-6}$ and $Q^{2}=2.4 \mathrm{GeV}^{2}$.) Figure 7 shows these parameterizations for $x G_{p}\left(x, Q^{2}\right)$ as a function of $x$ at $Q^{2}=2.4 \mathrm{GeV}^{2}$ (left panel) and at $Q^{2}=3 \mathrm{GeV}^{2}$ (right panel).
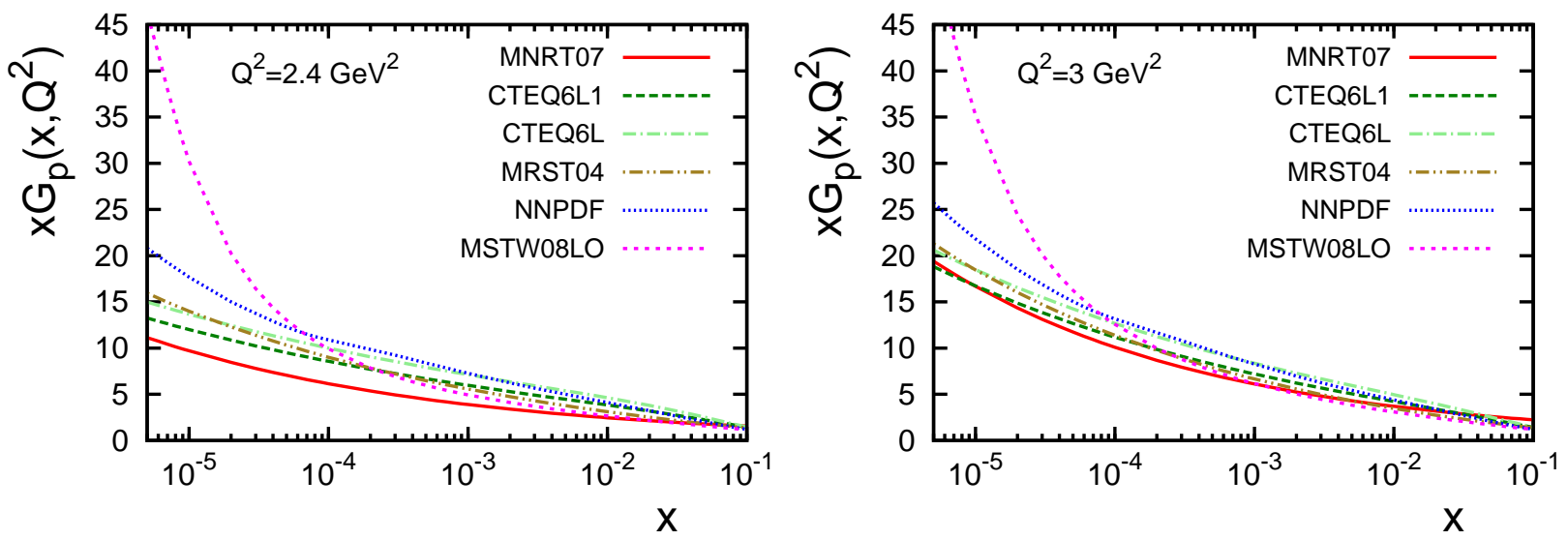

Figure 7. Leading order gluon parton distributions in the nucleon at $Q^{2}=2.4 \mathrm{GeV}^{2}$ (left) and at $Q^{2}=3 \mathrm{GeV}^{2}$ (right) used in this work.

Table 2 summarizes the values of strong coupling constant $\alpha_{s}\left(\mu^{2}\right)$ corresponding to the examined gluon parton distributions, which we used in our numerical predictions using eqs. (2.5), (2.6), and (2.12).

\begin{tabular}{|c|c|c|c|c|}
\hline Parameterization & $\alpha_{s}\left(Q^{2}=2.4 \mathrm{GeV}^{2}\right)$ & $\alpha_{s}\left(Q^{2}=3 \mathrm{GeV}^{2}\right)$ & $\alpha_{s}\left(M_{Z}^{2}\right)$ & Comments \\
\hline MNRT07 [14] & 0.295 & 0.282 & 0.118 & $\Lambda_{4}=120 \mathrm{MeV}$ \\
CTEQ6L1 [28] & 0.382 & 0.361 & 0.130 & $\Lambda_{4}=215 \mathrm{MeV}$ \\
CTEQ6L [28] & 0.330 & 0.314 & 0.118 & NLO with $\Lambda_{4}=326 \mathrm{MeV}$ \\
MRST04 [29] & 0.386 & 0.365 & - & $\Lambda_{4}=220 \mathrm{MeV}$ \\
NNPDF [30] & 0.301 & 0.289 & 0.119 & $\Lambda_{4}=127 \mathrm{MeV}$ \\
MSTW08LO [31] & 0.480 & 0.448 & 0.139 & $\Lambda_{4}=322 \mathrm{MeV}$ \\
\hline
\end{tabular}

Table 2. The running strong coupling constant $\alpha_{s}\left(\mu^{2}\right)$ corresponding to the examined gluon parton distributions.

Figure 8 shows the ratios of the studied gluon distributions of the proton to the MNRT07 gluon distribution as a function of $x$ at $Q^{2}=3 \mathrm{GeV}^{2}$. The ratios are normalized to be equal to unity at $x=10^{-3}$.

We explained in section 2.1 that to evaluate the factors of $\eta$ (the ratio of the real to the imaginary parts of the $\gamma p \rightarrow J / \psi p$ amplitude) and $R_{g}$ (the skewness factor), we used 


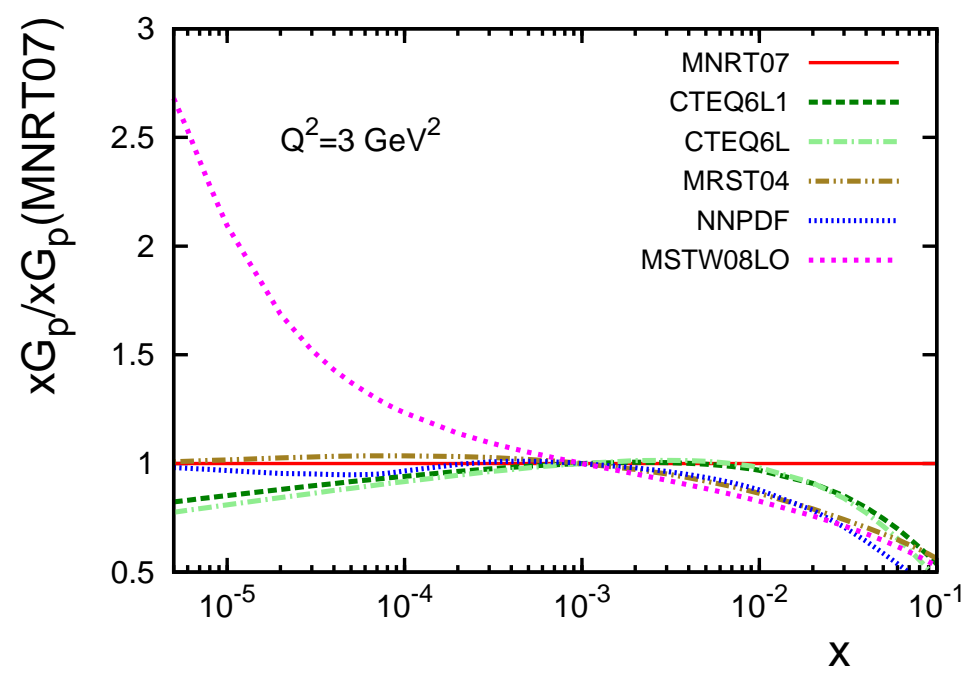

Figure 8. The ratios of the studied LO gluon distributions of the proton to the MNRT07 gluon distribution as a function of $x$ at $Q^{2}=3 \mathrm{GeV}^{2}$. The ratios are normalized to be equal to unity at $x=10^{-3}$.

the fit $x G_{p}\left(x, \mu^{2}\right) \propto 1 / x^{\lambda}$ in the interval $5 \times 10^{-6} \leq x \leq 0.01$. The resulting values of $\lambda$ are summarized in table 3 .

Note that the MSTW08LO gluon distribution cannot be fitted using a single $\lambda$ in the entire $5 \times 10^{-6} \leq x \leq 0.01$ interval. To achieve an acceptable accuracy of the $x G_{p}\left(x, \mu^{2}\right) \propto$ $1 / x^{\lambda}$ fit, we allowed $\lambda$ to vary with $x$ : the last line of table 3 gives the range of obtained values; $\lambda$ decreases with an increase of $x$.

\begin{tabular}{|c|c|c|}
\hline Parameterization & $\lambda\left(Q^{2}=2.4 \mathrm{GeV}^{2}\right)$ & $\lambda\left(Q^{2}=3 \mathrm{GeV}^{2}\right)$ \\
\hline MNRT07 [14] & 0.20 & 0.22 \\
CTEQ6L1 [28] & 0.16 & 0.19 \\
CTEQ6L [28] & 0.15 & 0.18 \\
MRST04 [29] & 0.20 & 0.22 \\
NNPDF [30] & 0.20 & 0.22 \\
MSTW08LO [31] & $0.62-0.30$ & $0.58-0.30$ \\
\hline
\end{tabular}

Table 3. The parameter $\lambda$ in the fit $x G_{p}\left(x, \mu^{2}\right) \propto 1 / x^{\lambda}$ of the studied proton gluon distributions in the interval $5 \times 10^{-6} \leq x \leq 0.01$ at $\mu^{2}=2.4 \mathrm{GeV}^{2}$ and $\mu^{2}=3 \mathrm{GeV}^{2}$. 


\section{References}

[1] R. Aaij et al. [LHCb Collaboration], Exclusive $J / \psi$ and $\psi(2 S)$ production in $p p$ collisions at $\sqrt{s}=7$ TeV, arXiv:1301.7084 [hep-ex].

[2] B. Abelev et al. [ALICE Collaboration], Coherent $J / \psi$ photoproduction in ultra-peripheral $\mathrm{Pb}-\mathrm{Pb}$ collisions at $\sqrt{s_{N N}}=2.76 \mathrm{TeV}$, Phys. Lett. B 718 (2013) 1273 [arXiv:1209.3715 [nucl-ex]].

[3] E. Abbas et al. [ALICE Collaboration], Charmonium and e+e-pair photoproduction at mid-rapidity in ultra-peripheral $\mathrm{Pb}-\mathrm{Pb}$ collisions at $\operatorname{sqrt}(\mathrm{sNN})=2.76 \mathrm{TeV}$, arXiv:1305.1467 [nucl-ex].

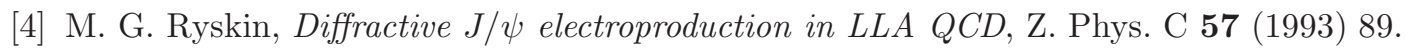

[5] S. J. Brodsky, L. Frankfurt, J. F. Gunion, A. H. Mueller and M. Strikman, Diffractive leptoproduction of vector mesons in QCD, Phys. Rev. D 50 (1994) 3134.

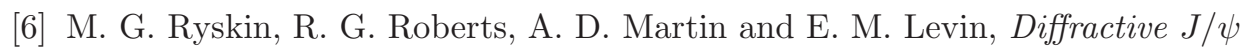
photoproduction as a probe of the gluon density, Z. Phys. C 76 (1997) 231 [hep-ph/9511228].

[7] L. Frankfurt, W. Koepf and M. Strikman, Hard diffractive electroproduction of vector mesons in QCD, Phys. Rev. D 54 (1996) 3194 [hep-ph/9509311].

[8] V. Rebyakova, M. Strikman and M. Zhalov, Coherent rho and J/psi photoproduction in ultraperipheral processes with electromagnetic dissociation of heavy ions at RHIC and LHC, Phys. Lett. B 710 (2012) 647 [arXiv:1109.0737 [hep-ph]].

[9] A. Adeluyi and C. A. Bertulani, Constraining Gluon Shadowing Using Photoproduction in Ultraperipheral $p A$ and AA Collisions, Phys. Rev. C 85 (2012) 044904 [arXiv:1201.0146 [nucl-th]].

[10] V. Guzey, E. Kryshen, M. Strikman and M. Zhalov, Evidence for nuclear gluon shadowing from the ALICE measurements of $\mathrm{PbPb}$ ultraperipheral exclusive $\mathrm{J} / \psi$ production, Phys. Lett. B 726 (2013) 290 [arXiv:1305.1724 [hep-ph]].

[11] A. Baltz et al., The Physics of Ultraperipheral Collisions at the LHC, Phys. Rept. 458 (2008) 1 [arXiv:0706.3356 [nucl-ex]].

[12] L. Frankfurt, W. Koepf and M. Strikman, Diffractive heavy quarkonium photoproduction and electroproduction in QCD, Phys. Rev. D 57 (1998) 512 [hep-ph/9702216].

[13] V. N. Gribov and A. A. Migdal, Properties of the pomeranchuk pole and the branch cuts related to it at low momentum transfer, Sov. J. Nucl. Phys. 8 (1969) 583 [Yad. Fiz. 8 (1968) 1002].

[14] A. D. Martin, C. Nockles, M. G. Ryskin and T. Teubner, Small x gluon from exclusive J/ $\psi$ production, Phys. Lett. B 662 (2008) 252 [arXiv:0709.4406 [hep-ph]].

[15] J. C. Collins, L. Frankfurt and M. Strikman, Factorization for hard exclusive electroproduction of mesons in QCD, Phys. Rev. D 56 (1997) 2982 [hep-ph/9611433].

[16] J. C. Collins and A. Freund, Proof of factorization for deeply virtual Compton scattering in QCD, Phys. Rev. D 59 (1999) 074009 [hep-ph/9801262].

[17] A. G. Shuvaev, K. J. Golec-Biernat, A. D. Martin and M. G. Ryskin, Off diagonal distributions fixed by diagonal partons at small $x$ and $\xi$, Phys. Rev. D 60 (1999) 014015 [hep-ph/9902410]. 
[18] L. A. Harland-Lang, A simple form for the low-x generalized parton distributions in the skewed regime, arXiv:1306.6661 [hep-ph].

[19] J. B. Bronzan, G. L. Kane and U. P. Sukhatme, "Obtaining Real Parts of Scattering Amplitudes Directly from Cross-Section Data Using Derivative Analyticity Relations, Phys. Lett. B 49 (1974) 272.

[20] L. Frankfurt, M. McDermott and M. Strikman, A Fresh look at diffractive J / psi photoproduction at HERA, with predictions for THERA, JHEP 0103 (2001) 045 [hep-ph/0009086].

[21] P. Hoodbhoy, Wave function corrections and off forward gluon distributions in diffractive $J / \psi$ electroproduction, Phys. Rev. D 56 (1997) 388 [hep-ph/9611207].

[22] C. Adloff et al. [H1 Collaboration], Elastic photoproduction of $J / \psi$ and Upsilon mesons at HERA, Phys. Lett. B 483 (2000) 23 [hep-ex/0003020].

[23] S. Chekanov et al. [ZEUS Collaboration], Exclusive photoproduction of $J / \psi$ mesons at HERA, Eur. Phys. J. C 24 (2002) 345 [hep-ex/0201043].

[24] A. Aktas et al. [H1 Collaboration], Elastic J/psi production at HERA, Eur. Phys. J. C 46 (2006) 585 [hep-ex/0510016].

[25] C. Alexa et al. [H1 Collaboration], Elastic and Proton-Dissociative Photoproduction of $J / \psi$ Mesons at HERA, arXiv:1304.5162 [hep-ex].

[26] L. Frankfurt, V. Guzey, M. Strikman and M. Zhalov, Onset of perturbative color opacity at small $x$ and Upsilon coherent photoproduction off heavy nuclei at LHC, JHEP 0308 (2003) 043 [hep-ph/0304218].

[27] M. Strikman, M. Tverskoy and M. Zhalov, Neutron tagging of quasielastic J/psi photoproduction off nucleus in ultraperipheral heavy ion collisions at RHIC energies, Phys. Lett. B 626 (2005) 72 [hep-ph/0505023].

[28] J. Pumplin, D. R. Stump, J. Huston, H. L. Lai, P. M. Nadolsky and W. K. Tung, New generation of parton distributions with uncertainties from global QCD analysis, JHEP 0207 (2002) 012 [hep-ph/0201195].

[29] A. D. Martin, W. J. Stirling and R. S. Thorne, MRST partons generated in a fixed-flavor scheme, Phys. Lett. B 636 (2006) 259 [hep-ph/0603143].

[30] R. D. Ball, L. Del Debbio, S. Forte, A. Guffanti, J. I. Latorre, J. Rojo and M. Ubiali, A first unbiased global NLO determination of parton distributions and their uncertainties, Nucl. Phys. B 838 (2010) 136 [arXiv:1002.4407 [hep-ph]]; http://nnpdf .hepforge.org.

[31] A. D. Martin, W. J. Stirling, R. S. Thorne and G. Watt, Parton distributions for the LHC, Eur. Phys. J. C 63 (2009) 189 [arXiv:0901.0002 [hep-ph]].

[32] L. Frankfurt, V. Guzey and M. Strikman, Leading Twist Nuclear Shadowing Phenomena in Hard Processes with Nuclei, Phys. Rept. 512 (2012) 255 [arXiv:1106.2091 [hep-ph]].

[33] K. J. Eskola, H. Paukkunen and C. A. Salgado, EPS09: A New Generation of NLO and LO Nuclear Parton Distribution Functions, JHEP 0904 (2009) 065 [arXiv:0902.4154 [hep-ph]].

[34] M. Hirai, S. Kumano and T. -H. Nagai, Determination of nuclear parton distribution 
functions and their uncertainties in next-to-leading order, Phys. Rev. C 76 (2007) 065207 [arXiv:0709.3038 [hep-ph]].

[35] D. de Florian and R. Sassot, Nuclear parton distributions at next-to-leading order, Phys. Rev. D 69 (2004) 074028 [hep-ph/0311227].

[36] D. de Florian, R. Sassot, P. Zurita and M. Stratmann, Global Analysis of Nuclear Parton Distributions, Phys. Rev. D 85 (2012) 074028 [arXiv:1112.6324 [hep-ph]].

[37] A. Adeluyi and C. Bertulani, Gluon distributions in nuclei probed at the CERN Large Hadron Collider, Phys. Rev. C 84 (2011) 024916 [arXiv:1104.4287 [nucl-th]].

[38] A. Adeluyi, C. A. Bertulani and M. J. Murray, Nuclear effects in photoproduction of heavy quarks and vector mesons in ultraperipheral $\mathrm{PbPb}$ and $\mathrm{pPb}$ collisions at the $\mathrm{LHC}$, Phys. Rev. C 86 (2012) 047901 [arXiv: 1208.6258 [nucl-th]].

[39] A. Adeluyi and T. Nguyen, Coherent photoproduction of $\psi$ and $\Upsilon$ mesons in ultraperipheral $p P b$ and $\mathrm{PbPb}$ collisions at the CERN Large Hadron Collider at $\sqrt{S_{N N}}=5 \mathrm{TeV}$ and $\sqrt{S_{N N}}=2.76 \mathrm{TeV}$, Phys. Rev. C 87 (2013) 027901 [arXiv:1302.4288 [nucl-th]].

[40] A. D. Martin, R. G. Roberts, W. J. Stirling and R. S. Thorne, Parton distributions: A New global analysis, Eur. Phys. J. C 4 (1998) 463 [hep-ph/9803445].

[41] V. P. Goncalves and M. V. T. Machado, Vector Meson Production in Coherent Hadronic Interactions: An update on predictions for RHIC and LHC, Phys. Rev. C 84 (2011) 011902 [arXiv:1106.3036 [hep-ph]].

[42] T. Lappi and H. Mantysaari, $J / P$ si production in ultraperipheral $P b+P b$ and $p+P b$ collisions at LHC energies, Phys. Rev. C 87 (2013) 032201 [arXiv:1301.4095 [hep-ph]].

[43] A. Cisek, W. Schafer and A. Szczurek, Exclusive coherent production of heavy vector mesons in nucleus-nucleus collisions at LHC, Phys. Rev. C 86 (2012) 014905 [arXiv:1204.5381 [hep-ph]].

[44] V. Guzey and M. Zhalov, Rapidity and momentum transfer distributions of coherent $J / \psi$ photoproduction in ultraperipheral $\mathrm{pPb}$ collisions at the LHC, arXiv:1307.6689 [hep-ph]. 